\title{
CONVOLUTION PROPERTIES OF A CLASS OF STARLIKE FUNCTIONS
}

\author{
RAM SINGH AND SUKHJIT SINGH
}

(Communicated by Paul S. Muhly)

\begin{abstract}
Let $R$ denote the class of functions $f(z)=z+a_{2} z^{2}+\cdots$ that are analytic in the unit disc $E=\{z:|z|<1\}$ and satisfy the condition $\operatorname{Re}\left(f^{\prime}(z)+\right.$ $\left.z f^{\prime \prime}(z)\right)>0, z \in E$. It is known that $R$ is a subclass of $S_{t}$, the class of univalent starlike functions in $E$. In the present paper, among other things, we prove (i) for every $n \geq 1$, the $n$th partial sum of $f \in R, s_{n}(z, f)$, is univalent in $E$, (ii) $R$ is closed with respect to Hadamard convolution, and (iii) the Hadamard convolution of any two members of $R$ is a convex function in $E$.
\end{abstract}

\section{INTRODUCTION}

Let $A$ denote the class of functions $f$ that are regular in the unit disc $E=$ $\{z:|z|<1\}$ and satisfy the conditions $f(0)=f^{\prime}(0)-1=0$. We denote by $S$ the subclass of $A$ consisting of univalent functions and by $K, S_{t}$, and $C$ the usual subclasses of $S$ whose members are convex, starlike (w.r.t. the origin) and close-to-convex, respectively. Finally, denote by $R$ the family of functions $f \in A$ which satisfy the condition $\operatorname{Re}\left(f^{\prime}(z)+z f^{\prime \prime}(z)\right)>0, z \in E$. Chichra [1] proved that if $f \in R$, then $\operatorname{Re} f^{\prime}(z)>0, z \in E$, and hence $f$ is univalent in $E$. R. Singh and S. Singh [8] showed that if $f \in R$ then $f$ is also starlike in $E$.

In the present paper we improve Chichra's result and show that the assertion of Singh and Singh holds under a much weaker hypothesis. We also prove that for every integer $n \geq 1$, the $n$th partial sum of $f \in R, s_{n}(z, f)$, is close-toconvex in $E$. Finally, we prove that $R$ is closed with respect to Hadamard convolution and that if $f, g \in R$, then their Hadamard convolution is convex in $E$. The significance of the last two results will be made clear later on at the appropriate place.

Received by the editors October 27, 1987 and, in revised form August 25, 1988.

1980 Mathematics Subject Classification (1985 Revision). Primary 30A26; Secondary 30A36, $30 \mathrm{~A} 42$.

Key words and phrases. Univalent, starlike and convex functions, convex null sequences. 


\section{Preliminaries}

We shall need the following definitions and results. If $f(z)=\sum_{n=0}^{\infty} a_{n} z^{n}$ and $g(z)=\sum_{n=0}^{\infty} b_{n} z^{n}$ are analytic in $|z|<\rho$, then their Hadamard product/convolution, $f * g$, is the function defined by the power series

$$
(f * g)(z)=\sum_{n=0}^{\infty} a_{n} b_{n} z^{n}
$$

The function $f * g$ is also analytic in $|z|<\rho$.

A sequence $\left\{c_{n}\right\}_{0}^{\infty}$ of non-negative numbers is said to be a convex null sequence if $c_{n} \rightarrow 0$ as $n \rightarrow \infty$ and

$$
c_{0}-c_{1} \geq c_{1}-c_{2} \geq \cdots \geq c_{n}-c_{n+1} \geq \cdots \geq 0 .
$$

If $f$ is analytic in $|z|<\rho, g$ is analytic and univalent in $|z|<\rho$ and $f(0)=g(0)$, then we say that $f$ is subordinate to $g$ in $|z|<\rho$, in symbols, $f \prec g$ in $|z|<\rho$, if $f(|z|<\rho) \subset g(|z|<\rho)$.

Lemma 1. Let $\left\{c_{n}\right\}_{0}^{\infty}$ be a convex null sequence. Then the function

$$
q(z)=\frac{c_{0}}{2}+\sum_{n=1}^{\infty} c_{n} z^{n}
$$

is analytic in $E$ and $\operatorname{Re} q(z)>0, z \in E$.

Lemma 2. Suppose that $w$ is a nonconstant function analytic in $|z|<\rho$ and $w(0)=0$. Then, if $|w(z)|$ attains its maximum value on the circle $|z|=r<\rho$ at the point $z_{0}$, we can write $z_{0} w^{\prime}\left(z_{0}\right)=k w\left(z_{0}\right)$, where $k \geq 1$.

Lemma 3. For $0 \leq \theta \leq \pi$,

$$
\frac{1}{2}+\sum_{k=1}^{n} \frac{\cos k \theta}{k+1} \geq 0
$$

Lemma 4. If $P(z)$ is analytic in $E, P(0)=1$, and $\operatorname{Re} P(z)>\frac{1}{2}, z \in E$, then for any function $F$, analytic in $E$, the function $P * F$ takes values in the convex hull of the image of $E$ under $F$.

Lemmas 1, 2, and 3 are due to Fejěr [2], Jack [3], and Rogosinski and Szegö [6], respectively. The assertion of Lemma 4 readily follows by using the Herglotz' representation for $P(z)$.

\section{THEOREMS AND THEIR PROOFS}

Theorem 1. Let $f \in R$. Then we have

(a) $\operatorname{Re} f^{\prime}(z)>-1+2 \log 2 \doteqdot 0 . \cdot 39 \ldots,(z \in E)$. The constant $-1+2 \log 2$ cannot be replaced by any larger one.

(b) $\operatorname{Re} \frac{f(z)}{z}>\frac{1}{2}, z \in E$.

(c) For every $n \geq 1$, the nth partial sum of $f, s_{n}(z, f)$, satisfies $\operatorname{Re} s_{n}^{\prime}(z, f)>0, z \in E$, and hence $s_{n}(z, f)$ is univalent in $E$. 
(d) For every $n \geq 1$

$$
\operatorname{Re} \frac{s_{n}(z, f)}{z}>\frac{1}{3} \quad(z \in E) .
$$

Proof. Let $f(z)=z+\sum_{n=2}^{\infty} a_{n} z^{n}$. Since $\operatorname{Re}\left(f^{\prime}(z)+z f^{\prime \prime}(z)\right)>0, z \in E$, we have

$$
\operatorname{Re}\left[1+\sum_{n=2}^{\infty} n^{2} a_{n} z^{n-1}\right]>0 \quad(z \in E)
$$

and hence

$$
\operatorname{Re}\left[1+\frac{1}{2} \sum_{n=2}^{\infty} n^{2} a_{n} z^{n-1}\right]>\frac{1}{2} \quad(z \in E) .
$$

Consider the function

$$
P(z)=1+2 \sum_{n=2}^{\infty} \frac{1}{n} z^{n-1} .
$$

Clearly $P(z)$ is analytic in $E, P(0)=1$ and

$$
\begin{aligned}
\operatorname{Re} P(z) & =\operatorname{Re}\left[-1-\frac{2}{z} \log (1-z)\right] \\
& >-1+2 \log 2[5] .
\end{aligned}
$$

Now, since we can write

$$
f^{\prime}(z)=\left[1+\frac{1}{2} \sum_{n=2}^{\infty} n^{2} a_{n} z^{n-1}\right] *\left[1+2 \sum_{n=2}^{\infty} \frac{1}{n} z^{n-1}\right],
$$

it follows, in view of (2), (3) and Lemma 4, that $\operatorname{Re} f^{\prime}(z)>-1+2 \log 2$, $z \in E$. That the constant $-1+2 \log 2$ cannot be replaced by any larger one follows from the fact that the function $f_{0}$ defined by $z f_{0}^{\prime}(z)=-z-2 \log (1-z)$ is in the class $R$.

(b) We observe that since the sequence $\left\{c_{n}\right\}_{0}^{\infty}$ defined by $c_{0}=1, c_{n}=$ $2 /(n+1)^{2}, n \geq 1$, is a convex null sequence, we have, in view of Lemma 1 .

$$
\operatorname{Re}\left[1+2 \sum_{n=2}^{\infty} \frac{1}{n^{2}} z^{n-1}\right]>\frac{1}{2} \quad(z \in E) .
$$

Writing $f(z) / z$ as

$$
\frac{f(z)}{z}=\left[1+\frac{1}{2} \sum_{n=2}^{\infty} n^{2} a_{n} z^{n-1}\right] *\left[1+2 \sum_{n=2}^{\infty} \frac{1}{n^{2}} z^{n-1}\right]
$$

and making use of (2), (4) and Lemma 4, we conclude that $\operatorname{Re}(f(z) / z)>\frac{1}{2}$, $z \in E$.

(c) We can write

$$
s_{n}^{\prime}(z, f)=\left[1+\sum_{k=2}^{\infty} k^{2} a_{k} z^{k-1}\right] *\left[1+\sum_{k=2}^{n} \frac{1}{k} z^{k-1}\right] .
$$


Putting $z=r e^{i \theta}, 0 \leq r<1,0 \leq|\theta| \leq \pi$, and making use of the minimum principle for harmonic functions along with Lemma 3, we obtain

$$
\begin{aligned}
\operatorname{Re}\left[1+\sum_{k=2}^{n} \frac{1}{k} z^{k-1}\right] & =\operatorname{Re}\left[1+\sum_{k=1}^{n-1} \frac{z^{k}}{k+1}\right] \\
& =1+\sum_{k=1}^{n-1} \frac{r^{k} \cos k \theta}{(k+1)} \quad(0 \leq \theta \leq \pi) \\
& >1+\sum_{k=1}^{n-1} \frac{\cos k \theta}{k+1} \\
& \geq \frac{1}{2} .
\end{aligned}
$$

In view of (1), (6), (5) and Lemma 4, we deduce that $\operatorname{Re} s_{n}^{\prime}(z, f)>0, z \in E$, and so $s_{n}(z, f)$ is close-to-convex in $E$ for every $n \geq 1$.

(d) Let

$$
q(z)=1+\sum_{k=2}^{n} \frac{1}{k} z^{k-1}
$$

Then by (6), we have $\operatorname{Re} q(z)>\frac{1}{2}$ in $E$. An application of Lemma 2 readily provides that

$$
\begin{aligned}
\operatorname{Re} S(z) & =\operatorname{Re}\left[\frac{1}{z} \int_{0}^{z} q(t) d t\right] \\
& =\operatorname{Re}\left[1+\sum_{k=2}^{n} \frac{1}{k^{2}} z^{k-1}\right]>\frac{2}{3} \quad(z \in E) .
\end{aligned}
$$

Writing $s_{n}(z, f) / z$ as

$$
\begin{aligned}
\frac{s_{n}(z, f)}{z} & =\left[1+\frac{1}{2} \sum_{k=2}^{\infty} k^{2} a_{k} z^{k-1}\right] *\left[1+2 \sum_{k=2}^{n} \frac{1}{k^{2}} k^{z-1}\right] \\
& =\left[1+\frac{1}{2} \sum_{k=2}^{\infty} k^{2} a_{k} z^{k-1}\right] *\left[-1+2\left(1+\sum_{k=2}^{n} \frac{1}{k^{2}} z^{k-1}\right)\right],
\end{aligned}
$$

and making use of (2), (7) and Lemma 4, the conclusion (d) follows at once.

Remark 1. It is clear that $f \in R$ if and only if $\operatorname{Re} g^{\prime}(z)>0, z \in E$, where $g(z)=z f^{\prime}(z)$. From this it follows that if $f \in R$, then $f$ has the integral representation

$$
f^{\prime}(z)=-1-\int_{|x|=1} \frac{2}{x z} \log (1-x z) d \mu(x),
$$

where $\mu$ is a probability measure on $|x|=1$.

Since $\operatorname{Re}[(-2 / x z) \log (1-x z)]>2 \log 2$ [5], part (a) also follows from (8).

If $f \in R$, then as seen in Theorem 1, part (c), each $s_{n}(z, f)$ is univalent in $E$. It is, therefore, natural to ask for the largest number $\lambda_{n}, 0<\lambda_{n}<1$, 
such that $\lambda_{n} s_{n}(z, f) \prec s_{n+1}(z, f), z \in E$. Using part (d) of the above theorem along with the fact that if $f(z)=z+\sum_{n=2}^{\infty} a_{n} z^{n} \in R$, then $\left|a_{n}\right| \leq 2 / n^{2}, n \geq 2$, we readily obtain the following result which provides a lower bound for $\lambda_{n}$.

Corollary 1. Let $f \in R$. Then

(i) $\frac{1}{2} z=\frac{1}{2} s_{1}(z, f) \prec s_{2}(z, f), \quad z \in E$,

(ii) $\frac{(n+1)^{2}-6}{(n+1)^{2}} s_{n}(z, f) \prec s_{n+1}(z, f), \quad z \in E, n \geq 2$.

The constant $\frac{1}{2}$ in (i) is the best possible one.

Our next result shows that the assertion of R. Singh and S. Singh mentioned in the Introduction holds under a much weaker hypothesis.

Theorenm 2. If $f \in A$ and

$$
\operatorname{Re}\left(f^{\prime}(z)+z f^{\prime \prime}(z)\right)>-\frac{1}{4} \quad(z \in E),
$$

then $f \in S_{t}$.

Proof. Letting $f(z)=z+\sum_{n=2}^{\infty} a_{n} z^{n}$, it follows from the hypothesis of the theorem that

$$
\operatorname{Re}\left[1+\frac{2}{5} \sum_{k=2}^{\infty} k^{2} a_{k} z^{k-1}\right]>\frac{1}{2}, \quad z \in E .
$$

Also one can easily see that the sequence $\left\{c_{n}\right\}_{0}^{\infty}$, where $c_{0}=1$ and $c_{n}=$ $5 / 2(n+1)^{2}, n \geq 1$, is a convex null sequence and as such

$$
\operatorname{Re}\left[1+\frac{5}{2} \sum_{k=2}^{\infty} \frac{1}{k^{2}} z^{k-1}\right]>\frac{1}{2} \quad(z \in E) .
$$

From (9) and (10) and Lemma 4 we deduce that

$$
\begin{aligned}
\operatorname{Re} \frac{f(z)}{z} & =\operatorname{Re}\left[\left(1+\frac{2}{5} \sum_{k=2}^{\infty} k^{2} a_{k} z^{k-1}\right) *\left(1+\frac{5}{2} \sum_{k=2}^{\infty} \frac{1}{k^{2}} z^{k-1}\right)\right] \\
& >\frac{1}{2}, \quad z \in E .
\end{aligned}
$$

An application of Lemma 2 readily yields that if $f$ satisfies the hypothesis of Theorem 2, then $\operatorname{Re} f^{\prime}(z)>0, z \in E$, and hence $f$ is univalent in $E$. Define a function $w$ in $E$ by

$$
\frac{z f^{\prime}(z)}{f(z)}=\frac{1+w(z)}{1-w(z)}
$$

Clearly $w$ so defined is meromorphic in $E, w(0)=0$ and since $f$ is univalent in $E$, we have $w(z) \neq 1$ in $E$. From (12) we obtain

$$
f^{\prime}(z)+z f^{\prime \prime}(z)=\left(\frac{f(z)}{z}\right)\left[\left(\frac{1+w(z)}{1-w(z)}\right)^{2}+\frac{2 z w^{\prime}(z)}{(1-w(z))^{2}}\right] \text {. }
$$


We claim that $|w(z)|<1$ in $E$. If possible, suppose that there exists a point $z_{0} \in E$ such that $\max _{|z| \leq\left|z_{0}\right|}|w(z)|=\left|w\left(z_{0}\right)\right|=1$. Then from Lemma 2 it follows that $z w^{\prime}\left(z_{0}\right)=k w\left(z_{0}\right)$, where $k \geq 1$ and $w\left(z_{0}\right)=e^{i \theta}, 0<\theta<2 \pi$. Putting $z=z_{0}$ in (13), we get

$$
\begin{aligned}
\operatorname{Re}\left[f^{\prime}\left(z_{0}\right)+z_{0} f^{\prime \prime}\left(z_{0}\right)\right] & =\operatorname{Re}\left[\left(\frac{f\left(z_{0}\right)}{z_{0}}\right)\left\{\left(\frac{1+e^{i \theta}}{\left(1-e^{i \theta}\right.}\right)^{2}+\frac{2 k e^{i \theta}}{\left(1-e^{i \theta}\right)^{2}}\right\}\right] \\
& \leq-\frac{k}{2 \sin ^{2}(\theta / 2)} \operatorname{Re}\left(\frac{f\left(z_{0}\right)}{z_{0}}\right) \\
& \leq-\frac{1}{4}
\end{aligned}
$$

since $k \geq 1$ and, in view of (11), $\operatorname{Re}(f(z) / z)>\frac{1}{2}, z \in E$. As (14) contradicts our hypothesis, we conclude that $|w(z)|<1$ in $E$. Equation (12) then implies that $f$ must belong to $S_{t}$.

Corollary 2. If $g \in A$ and

$$
\operatorname{Re}\left[g^{\prime}(z)+3 z g^{\prime \prime}(z)+z^{2} g^{\prime \prime \prime}(z)\right]>-\frac{1}{4} \quad(z \in E),
$$

then $g \in K$.

It is known [7] that if $f \in S_{t}$ and $g \in K$, then $f * g \in S_{t}$ and that if $f$, $g \in S_{t}$, then $f * g$ need not be in $S_{t}$. In the following theorem we prove that if $f, g \in R$, a subclass of $S_{t}$, then so does $f * g$, i.e. $R$ is closed with respect to Hadamard product.

Theorem 3. If $f(z)=z+\sum_{n=2}^{\infty} a_{n} z^{n}$ and $g(z)=z+\sum_{n=2}^{\infty} b_{n} z^{n}$ belong to $R$, then so does their Hadamard product

$$
h(z)=(f * g)(z)=z+\sum_{n=2}^{\infty} a_{n} b_{n} z^{n} .
$$

Proof. Since $h(z)=(f * g)(z)$, we have

$$
z h^{\prime}(z)=z f^{\prime}(z) * g(z)
$$

and hence

$$
h^{\prime}(z)+z h^{\prime \prime}(z)=\left(f^{\prime}(z)+z f^{\prime \prime}(z)\right) * g(z) / z .
$$

Since $\operatorname{Re}\left(f^{\prime}(z)+z f^{\prime \prime}(z)\right)>0, z \in E$, and by Theorem 1, part (b), $\operatorname{Re}(g(z) / z)>\frac{1}{2}, z \in E$, the desired result follows at once from (15) and Lemma 4.

From the proof of Theorem 3 it is clear that in fact the following more general result holds:

Theorem $3^{\prime}$. If $f \in R, g \in A$ and $\operatorname{Re}(g(z) / z)>\frac{1}{2}, z \in E$, then $f * g \in R$.

We observe that $\operatorname{Re}(g(z) / z)>\frac{1}{2}, z \in E$, need not even imply the univalence of $g$ in $E$. 
Corollary 3. If $f(z)=z+\sum_{n=2}^{\infty} a_{n} z^{n} \in R$, then so does

$$
f_{k}(z)=z+\sum_{n=1}^{\infty} a_{n k+1} z^{n k+1}, \quad k=1,2,3, \ldots
$$

In the next theorem we prove that if $f, g \in R$, then $f * g \in K$. Since the class $K$ is closed with respect to Hadamard convolution [7], the significance of our result will be apparent only if we show that $R$ (which has hitherto been shown to be a subset of $S_{t}$ ) is not contained in $K$. To prove that $R \nsubseteq$ $K$, denote by $P^{\prime}$ the family of functions $f \in A$ which satisfy the condition $\operatorname{Re} f^{\prime}(z)>0, z \in E$. Krzyż [4] has demonstrated a function $f_{0} \in P^{\prime}$ such that $f_{0} \notin S_{t}$ (space does not permit us to carry out the construction of $f_{0}$ ). Clearly the function $f^{*}$, defined by $f^{*}(z)=\int_{0}^{z}\left(f_{0}(\zeta) / \zeta\right) d \zeta$, is a member of $R$ which is not in $K$, showing that $R \nsubseteq K$.

Theorem 4. If $f(z)=z+\sum_{n=2}^{\infty} a_{n} z^{n}$ and $g(z)=z+\sum_{n=2}^{\infty} b_{n} z^{n}$ are in $R$, then

$$
h(z)=(f * g)(z)=z+\sum_{n=2}^{\infty} a_{n} b_{n} z^{n} \in K .
$$

Proof. In view of Corollary 2 it suffices to show that

$$
\operatorname{Re}\left[h^{\prime}(z)+3 z h^{\prime \prime}(z)+z^{2} h^{\prime \prime \prime}(z)\right]>-\frac{1}{4}, \quad z \in E,
$$

or, equivalently,

$$
\operatorname{Re}\left[1+\sum_{n=2}^{\infty} n^{3} a_{n} b_{n} z^{n-1}\right]>-\frac{1}{4}, \quad z \in E .
$$

Since $f, g \in R$, we have

$$
\operatorname{Re}\left[1+\frac{1}{2} \sum_{n=2}^{\infty} n^{2} a_{n} z^{n-1}\right]>\frac{1}{2}, \quad z \in E,
$$

and

$$
\operatorname{Re}\left[1+\frac{1}{2} \sum_{n=2}^{\infty} n^{2} b_{n} z^{n-1}\right]>\frac{1}{2}, \quad z \in E .
$$

Therefore, in view of Lemma 4, it follows that

$$
\operatorname{Re}\left[1+\frac{1}{4} \sum_{n=2}^{\infty} n^{4} a_{n} b_{n} z^{n-1}\right]>\frac{1}{2}, \quad z \in E .
$$

Now, we can write

$$
\left[1+\sum_{n=2}^{\infty} n^{3} a_{n} b_{n} z^{n-1}\right]=\left[1+\frac{1}{4} \sum_{n=2}^{\infty} n^{4} a_{n} b_{n} z^{n-1}\right] *\left[1+4 \sum_{n=2}^{\infty} \frac{1}{n} z^{n-1}\right] .
$$


Since

$$
\begin{aligned}
\operatorname{Re}\left[1+4 \sum_{n=2}^{\infty} \frac{1}{n} z^{n-1}\right] & =\operatorname{Re}\left[-3-\frac{4}{z} \log (1-z)\right] \\
& >-3+4 \log 2[5] \\
& \doteqdot-0.231>-\frac{1}{4} \quad(z \in E),
\end{aligned}
$$

it follows from (17), (18), (19) and Lemma 4 that (16) holds for all $z \in E$. The proof of Theorem 4 is, therefore, complete.

\section{REFERENCES}

1. P. N. Chichra, New subclasses of the class of close-to-convex functions, Proc. Amer. Math. Soc. (1) 62 (1977), 37-43.

2. L. Fejër, Uber die positivitat von summen, die nach trigonometrischen order Legendreschen funktionen fortschreiten, Acta Litt. Ac Sci. Szeged (1925), 75-86.

3. I. S. Jack, Functions starlike and convex of order $\alpha$, J. London Math Soc. (2) 3 (1971), 469474.

4. J. Krzyź, A counter example concerning univalent functions, Mat. Fiz. Chem. 2 (1962), 57-58.

5. M. S. Robertson, An extremal problem for functions with positive real part, Michigan Math. J. 11 (1964), 327-335.

6. W. Rogosinski and G. Szegö, Uber die Abschimlte Von potenzreihen die in ernein Kreise be schrankt bleiben, Math. Z., 28 (1928), 73-94.

7. St. Ruscheweyh and T. Sheil Small, Hadamard products of schlicht functions and the PolyaSchoenberg conjecture, Comm. Math. Helv. 48 (1973), 119-135.

8. R. Singh and S. Singh, Starlikeness and convexity of certain integrals, Ann. Univ. Mariae Curie-Skłodowska Sect. A 35 (1981), 45-47.

Department of Mathematics, Punjabi University, Patiala-147002 (Punjab) India 